\title{
Analyses of the Spurious Oscillations in the Lumped Parameters Line Model
}

\author{
A. R. J.de Araújo, R. C. da Silva and S. Kurokawa
}

\begin{abstract}
The currents and voltages in the multiphase transmission line are described by differential equations that sometimes can be difficult to solve due the mutual coupling and skin effect that have to be considered for accurate results. An alternative consists in using a matrix to decompose the multiphase transmission line in $n$ single-phase systems uncoupled. For evaluating modal voltages and currents, each system will be represented by two models well-known for studies in electromagnetic transients in transmission line. The first model uses the lumped parameters to represent the transmission line and it is developed directly in the time domain. The second model uses the distributed parameters and transforms the differential equations in the time domain to algebraic equations in the frequency domain using Laplace Transform. Once solved them, it can be applied the inverse transformation and the solutions are obtained in the time domain. It can be observed the presence of spurious oscillations in the lumped model simulations. This work will analyze the spurious oscillations in the voltages in the open receiving end using, considering a fixed-length transmission line, represented by different quantities of $\pi$-circuits during energization process.
\end{abstract}

Keywords - Time-Frequency domain, lumped and distributed parameters models, electromagnetics transients, model transformation.

\section{INTRODUÇÃO}

A S CORRENTES e tensões de uma linha de transmissão polifásica são descritas por equações diferenciais de difícil resolução devido ao seu acoplamento mútuo e aos efeitos pelicular e solo presentes. Uma alternativa para o estudo dos transitórios eletromagnéticos em sistemas de energia elétrica de $n$ fases consiste em decompô-lo em $n$ sistemas monofásicos desacoplados entre si, denominados de modos de propagação, e matematicamente idênticos ao sistema original. Cada modo de propagação é estudado separadamente, e uma vez obtidas as correntes e tensões no domínio modal, as correntes e tensões do sistema polifásico são calculadas utilizando matrizes de transformação [1]-[2];

Para a obtenção das correntes e tensões em cada modo, pode-se representar a linha de transmissão por diversos modelos. Neste trabalho cada linha de transmissão modal será representada por dois modelos: O primeiro modelo é conhecido como Modelo a Parâmetros Discretos que considera a linha representada por circuitos $\pi$ conectados em cascata e outro modelo utiliza os parâmetros distribuídos da

A. R. J. de Araujo, Universidade Estadual Paulista (UNESP), Ilha Solteira, São Paulo, Brasil, anderjusto@yahoo.com.br

R. C. da Silva, Instituto Federal de São Paulo,Campus de Votuporanga, São Paulo, Brasil, rcleber@gmail.com

S. Kurokawa, Universidade Estadual Paulista (UNESP), Ilha Solteira, São Paulo, Brasil, kurokawa@dee.feis.unesp.br. linha e as equações hiperbólicas da linha de transmissão, sendo denominado de Universal Line Model (ULM).

Tem-se reconhecido que um dos mais importantes aspectos na modelagem de linhas de transmissão para estudos em transitórios eletromagnéticos consiste em considerar a dependência da frequência nos parâmetros e perdas distribuídas [3]. Martí cita que modelos que consideram parâmetros constantes não são adequados para simular a resposta transitória para uma ampla faixa de frequências que estão presentes durante o transitório. Na maioria dos casos, usando a representação por parâmetros constantes, se produz sinais de elevadas harmônicas e como consequência, uma distorção geral do formato da onda do transitório com picos de exageradas magnitudes [3].

Nas respostas do modelo a parâmetros discretos são observadas oscilações numéricas denominadas (picos de exageradas magnitudes) que não correspondem ao valor real do transitório eletromagnético. Essas oscilações serão denominadas de que oscilações espúrias. Sabe que as oscilações espúrias dependem da quantidade de circuitos $\pi$ utilizada na representação da linha de transmissão, assim como o seu próprio comprimento. Neste trabalho serão mostradas as oscilações espúrias para simulações de uma linha de transmissão trifásica em aberto submetida a um processo de energização e serão feitas análises do seu conteúdo espectral considerando a linha representada por diferentes quantidades de circuitos $\pi$, verificando a sua influência nos resultados obtidos.

\section{TRANSFORMAÇÃO MODAL}

Para um sistema polifásico genérico, são utilizadas as matrizes impedância longitudinal por unidade de comprimento [Z] e a matriz de admitância transversal por unidade de comprimento $[\mathrm{Y}]$ da linha. Para o produto $[\mathrm{Z}][\mathrm{Y}]$ existem diversos conjuntos de autovetores que desacoplam a linha em seus modos exatos de propagação [3]. Os modos exatos são totalmente desacoplados entre si e são obtidos a partir da utilização das matrizes $\left[\mathrm{T}_{\mathrm{I}}\right]$ e $\left[\mathrm{T}_{\mathrm{V}}\right]$ como sendo as matrizes de transformação. As matrizes $\left[\mathrm{T}_{\mathrm{I}}\right]$ e $\left[\mathrm{T}_{\mathrm{V}}\right]$ são os autovetores associados aos produtos [Y][Z] e [Z][Y]. Outra possibilidade consiste em utilizar os "quase-modos" que são obtidos a partir do uso da matriz de Clarke [ $\left.\mathrm{T}_{\text {clarke }}\right]$ como sendo uma matriz de transformação. A [ $\left.\mathrm{T}_{\text {clarke }}\right]$ é uma matriz real e constante, cujos elementos são independentes da frequência, de fácil implementação em softwares que realizam simulações diretamente no domínio do tempo [4]. Se a linha de transmissão é idealmente transposta a [ $\left.\mathrm{T}_{\text {clarke }}\right]$ e decompõe a linha nos seus modos exatos. Quando a linha de transmissão é idealmente transposta, a [ $\left.\mathrm{T}_{\text {clarke }}\right]$ separa a linha em seus modos exatos. Em situações em que uma linha não pode ser considerada idealmente transposta, mas possui um plano de 
simetria vertical, pode-se, com algumas aproximações, utilizar a $\left[\mathrm{T}_{\text {clarke }}\right]$ para determinar os seus modos exatos. Nestas condições obtém-se a linha decomposta em seus quase-modos. Para linhas decompostas em seus quase-modos, as matrizes $\left[\mathrm{Y}_{\mathrm{qm}}\right]$ e $\left[\mathrm{Z}_{\mathrm{qm}}\right]$ possuem alguns elementos não nulos fora da diagonal principal, que serão desprezados e neste caso obtémse seus quase-modos [1].

Portanto, considerando a linha trifásica, transposta ou não, os modos exatos podem ser considerados equivalentes aos quase-modos, que serão denominados como alfa, beta e zero, respectivamente. A $\left[\mathrm{T}_{\text {clarke }}\right]$ é expressa como sendo conforme (1):

$$
\left[T_{\text {clarke }}\right]=\left[\begin{array}{ccc}
\frac{2}{\sqrt{6}} & 0 & \frac{2}{\sqrt{6}} \\
-\frac{1}{\sqrt{6}} & \frac{1}{\sqrt{2}} & \frac{1}{\sqrt{3}} \\
-\frac{1}{\sqrt{6}} & -\frac{1}{\sqrt{2}} & \frac{1}{\sqrt{3}}
\end{array}\right]
$$

As matrizes de impedâncias e admitâncias dos quasemodos da linha são expressas como mostram (2) e (3):

$$
\begin{aligned}
& {\left[Z_{q m}\right]=\left[T_{\text {clarke }}\right]^{T}[Z]\left[T_{\text {clarke }}\right]} \\
& {\left[Y_{q m}\right]=\left[T_{\text {clarke }}\right]^{-1}[Y]\left[T_{\text {clarke }}\right]^{-T}}
\end{aligned}
$$

Se a linha de transmissão é idealmente transposta, as matrizes $\left[\mathrm{Z}_{\mathrm{qm}}\right]$ e $\left[\mathrm{Y}_{\mathrm{qm}}\right]$ são idênticas às matrizes modais de impedância e admitância $\left[\mathrm{Z}_{\mathrm{m}}\right]$ e $\left[\mathrm{Y}_{\mathrm{m}}\right]$. Ou seja, nessas condições a $\left[\mathrm{T}_{\text {clarke }}\right]$ decompõe a linha em seus modos exatos. Caso a linha possua um plano de simetria vertical, mas não possa ser considerada idealmente transposta, as matrizes $\left[\mathrm{Z}_{\mathrm{qm}}\right]$ e [ $\left.\mathrm{Y}_{\mathrm{qm}}\right]$ são escritas como mostram (4) e (5):

$$
\begin{aligned}
& {\left[Z_{q m}\right]=\left[\begin{array}{ccc}
Z_{\alpha} & 0 & Z_{\alpha 0} \\
0 & Z_{\beta} & 0 \\
Z_{0 \alpha} & 0 & Z_{0}
\end{array}\right]} \\
& {\left[Y_{q m}\right]=\left[\begin{array}{ccc}
Y_{\alpha} & 0 & Y_{\alpha 0} \\
0 & Y_{\beta} & 0 \\
Y_{0 \alpha} & 0 & Y_{0}
\end{array}\right]}
\end{aligned}
$$

As equações (4) e (5) mostram que quando a linha não é idealmente transposta, existe acoplamento entre os quasemodos de propagação, que aqui são denominados de alfa, beta e zero. No entanto, em determinadas situações, o acoplamento entre os modos alfa, beta e zero pode ser desconsiderado. Nestas condições, as matrizes $\left[\mathrm{Z}_{\mathrm{qm}}\right]$ e $\left[\mathrm{Y}_{\mathrm{qm}}\right]$ tornam-se (5):

$$
\begin{aligned}
& {\left[Z_{q m}\right] \cong\left[\begin{array}{ccc}
Z_{\alpha} & 0 & 0 \\
0 & Z_{\beta} & 0 \\
0 & 0 & Z_{0}
\end{array}\right]} \\
& {\left[Y_{q m}\right] \cong\left[\begin{array}{ccc}
Y_{\alpha} & 0 & 0 \\
0 & Y_{\beta} & 0 \\
0 & 0 & Y_{0}
\end{array}\right]}
\end{aligned}
$$

As tensões dos quase-modos são obtidas conforme mostram (6).

$$
\left[V_{q m}\right]=\left[T_{\text {clarke }}\right]^{T}\left[V_{123}\right]
$$

Uma vez obtidos os valores de tensões nos modos alfa, beta e zero, as tensões trifásicas da linha são obtidas por (7).

$\left[V_{123}\right]=\left[T_{\text {clarke }}\right]^{-T}\left[V_{q m}\right]$

$\mathrm{Na}$ equação (7), o vetor $\left[\mathrm{V}_{123}\right]$ representa as tensões nas fases 1,2 e 3 da linha trifásica respectivamente obtida a partir da combinação linear das tensões nos modos alfa, beta e zero.

\section{SOLUÇÕES DAS EQUAÇÕES DIFERENCIAIS DA LINHA DE TRANSMISÃO}

As equações diferenciais que modelam uma linha polifásica são de difícil solução no domínio do tempo, porém no domínio da frequência estas equações são simples e suas soluções são conhecidas. A solução no domínio da frequência é genérica e pode ser aplicada para qualquer condição da linha, considerando os parâmetros fixos e/ou variáveis em função da frequência. Quanto à solução no domínio do tempo, essas são dependentes das integrais de convolução cujas soluções não são facilmente obtidas. Serão apresentados os modelos a parâmetros distribuídos e discretos e as soluções das equações da linha de transmissão no domínio do tempo e da frequência.

Considerando que a linha de transmissão trifásica foi decompostas em 3 linhas de transmissão monofásicas, serão descritas as equações das correntes e tensões que modelam uma linha monofásica para o modo de propagação genérico “ $n$ ” conforme a Fig. 1[4].

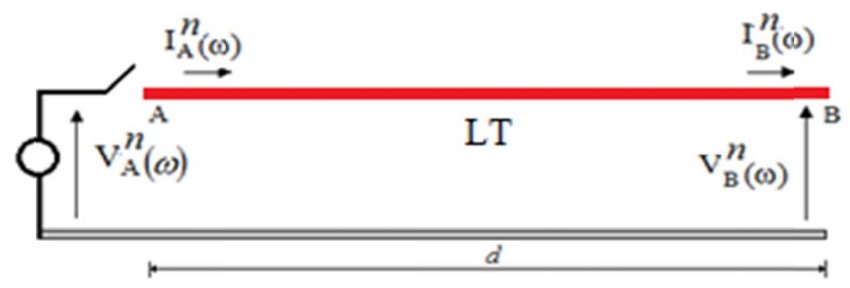

Figura 1. Linha de transmissão de comprimento d.

A linha mostrada na Fig. 1 possui uma impedância modal longitudinal e uma admitância transversal dada por (8):

$$
Z^{n}(\omega)=R^{n}+j \omega L^{n} \quad Y^{n}(\omega)=G^{n}+j \omega C^{n}
$$

Onde $\mathrm{R}$ e L são a resistência e a indutância longitudinais, $\mathrm{C}$ e $\mathrm{G}$ são a capacitância e a condutância transversais da linha por unidade de comprimento. Na Fig. $1 \mathrm{I}_{\mathrm{A}}(\omega)$ e $\mathrm{I}_{\mathrm{B}}(\omega)$ são as correntes nos terminais $\mathrm{A}$ e $\mathrm{B}$ da linha, enquanto que $\mathrm{V}_{\mathrm{A}}(\omega)$ e $\mathrm{V}_{\mathrm{B}}(\omega)$ são as tensões nestes terminais. As equações das correntes no domínio da frequência são dadas por:

$$
I_{A}{ }^{n}(\omega)=Y_{A A}^{n}(\omega) V_{A}^{n}(\omega)+Y_{A B}^{n}(\omega) V_{B}^{n}(\omega)
$$




$$
I_{B}^{n}(\omega)=Y_{B A}^{n}(\omega) V_{A}^{n}(\omega)+Y_{B B}^{n}(\omega) V_{B}^{n}(\omega)
$$

Onde os termos $\mathrm{Y}_{\mathrm{AA}}(\omega), \mathrm{Y}_{\mathrm{AB}}(\omega), \mathrm{Y}_{\mathrm{BA}}(\omega)$ e $\mathrm{Y}_{\mathrm{BB}}(\omega)$ são calculados por :

$$
\begin{aligned}
& Y_{B B}^{n}=Y_{A B}^{n}=-\frac{1}{Z^{n}{ }_{C}} \operatorname{csch}\left(\gamma^{n}(\omega) d\right) \\
& Y_{A A}^{n}=Y_{B A}^{n}=\frac{1}{Z^{n}{ }_{C}} \operatorname{coth}\left(\gamma^{n}(\omega) d\right)
\end{aligned}
$$

Em (11) $Z^{\mathrm{n}} c(\omega)$ e $\gamma^{\mathrm{n}}(\omega)$ são a impedância característica e a função de propagação da linha e são escritos como sendo:

$$
\begin{aligned}
& Z^{n}{ }_{C}(\omega)=\sqrt{\frac{Z^{n}(\omega)}{Y^{n}(\omega)}} \\
& \gamma^{n}(\omega)=\sqrt{Z^{n}(\omega) Y^{n}(\omega)}
\end{aligned}
$$

A transformação de (9) e (10) para o domínio do tempo não é facilmente obtida de modo analítico devido às integrais de convolução observadas. Uma alternativa consiste em transformar as equações diferenciais do domínio do tempo em equações algébricas para o domínio da frequência. Esse modelo considera que os parâmetros da linha são distribuídos ao longo do seu comprimento, e utiliza as equações algébricas hiperbólicas no domínio da frequência sendo denominado por Universal Line Model [5]. Uma vez encontradas as soluções, utilizando a Transformada Inversa de Laplace, são obtidas as respostas no domínio do tempo. A transformada de Laplace de uma função $\mathrm{f}(t)$ é definida como mostrado em (14):

$$
F(s)=L\{f(t)\}=\int_{0}^{\infty} f(t) e^{-s t} d t
$$

Aplicando a Transformada Inversa de Laplace em (9) e (10) e considerando que $\mathrm{s}=j \omega$ obtém-se:

$$
\begin{aligned}
I^{n}{ }_{A}(t) & =\frac{1}{2 \pi} \int_{-j \infty}^{+j \infty}\left[Y^{n}{ }_{A A}(\omega) V^{n}{ }_{A}(\omega)+Y^{n}{ }_{A B}(\omega) V^{n}{ }_{B}(\omega)\right] e^{j \omega t} d \omega \\
I^{n}{ }_{B}(t) & =\frac{1}{2 \pi} \int_{-j \infty}^{+j \infty}\left[Y_{B A}^{n}(\omega) V_{A}^{n}(\omega)+Y_{B B}^{n}(\omega) V_{B}^{n}(\omega)\right] e^{j \omega t} d \omega
\end{aligned}
$$

Geralmente essas equações são de difícil resolução sendo que não é possível determinar uma solução analítica para as grandezas $\mathrm{I}_{\mathrm{A}}(t), \quad \mathrm{I}_{\mathrm{B}}(t), \quad \mathrm{V}_{\mathrm{A}}(t)$ e $\mathrm{V}_{\mathrm{B}}(t)$ [10]. Portanto, são utilizados métodos numéricos que resolvem (9) e (10) no domínio da frequência e utilizando a Transformada Inversa de Laplace implementada numericamente, conforme o algoritmo proposto em $[6,11]$, pode-se obter a resposta no domínio do tempo.

\section{MODELO A PARÂMETROS DISCRETOS}

O modelo a parâmetros discretos é muito utilizado na representação de linhas de transmissão para o estudo de transitórios eletromagnéticos. Nesse modelo a linha é representada por circuitos $\pi$ conectados em cascata e podemse considerar as perdas, o efeito da frequência e o efeito corona. Será mostrada uma linha de transmissão monofásica representada por uma cascata de circuitos $\pi$, bem como as técnicas de obtenção das correntes e tensões na linha utilizando o modelo proposto.

Uma linha de transmissão monofásica poder ser representada por parâmetros discretos, como sendo uma cascata de $n$ circuitos $\pi$ [12] conforme mostra a Fig. 2 .

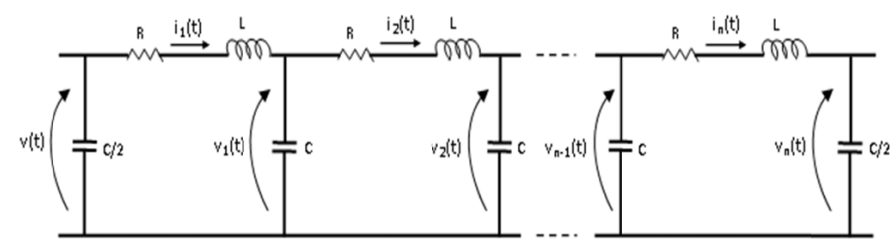

Figura 2. Linha representada por cascatas de circuitos $\pi$.

Na Fig. 2, os parâmetros $\mathrm{R}$ e $\mathrm{L}$ são, respectivamente, a resistência e a indutância longitudinais de cada segmento de linha. Os parâmetros G e C são, respectivamente, a condutância e a capacitância do segmento de linha. Os parâmetros R, L, G e C são escritos como sendo:

$R=\mathrm{R}^{\prime} \frac{\mathrm{d}}{\mathrm{n}} \quad L=\mathrm{L}^{\prime} \frac{\mathrm{d}}{\mathrm{n}} \quad C=\mathrm{C}^{\prime} \frac{\mathrm{d}}{\mathrm{n}} \quad G=\mathrm{G}^{\prime} \frac{\mathrm{d}}{\mathrm{n}}$

Em (17) os termos R' e L' são os parâmetros longitudinais da linha por unidade de comprimento e os termos e $C^{\prime}$ e $G^{\prime}$ são os parâmetros transversais da linha por unidade de comprimento. O modelo a parâmetros discretos mostrado na Fig. 2 fornece as correntes e tensões da linha diretamente no domínio do tempo e podem ser facilmente implementado em qualquer linguagem computacional [7]-[8]. Considere uma linha de transmissão de comprimento $d$, representada por $n$ circuitos $\pi$, como mostra a Fig 2 . As correntes longitudinais e as tensões transversais da linha podem ser escritas na forma de equações de estado.

$$
\begin{aligned}
& {[\dot{x}]=[A][x]+[B] u(t)} \\
& {[\dot{x}]=\left[\begin{array}{lllllll}
\dot{i}_{1}(t) & \dot{v}_{1}(t) & \dot{i}_{2}(t) & \dot{v}_{2}(t) & \ldots & \dot{i}_{n}(t) & \dot{v}_{n}(t)
\end{array}\right]^{T}}
\end{aligned}
$$

Em (18), [x] é um vetor com as correntes e tensões em cada um dos circuitos $\pi$, enquanto que [A] e [B] são matrizes de estados da cascata. Para uma linha de transmissão representada por $n$ circuitos $\pi$ conectados em cascata, as matrizes $[\mathrm{A}]$ e $[\mathrm{B}]$ são dadas por:

$$
\begin{aligned}
& {[A]=\left[\begin{array}{cccccc}
-R / L & -1 / L & 0 & 0 & 0 & 0 \\
1 / C & -G / C & -1 / C & 0 & 0 & 0 \\
0 & \ddots & \ddots & \ddots & 0 & 0 \\
0 & 0 & \ddots & \ddots & \ddots & 0 \\
0 & 0 & 0 & 1 / C & -G / C & -1 / C \\
0 & 0 & 0 & 0 & 1 / L & -R / L
\end{array}\right]} \\
& {[B]=\left[\begin{array}{llllll}
\frac{1}{L} & 0 & 0 & \cdots & 0 & 0
\end{array}\right]^{T}}
\end{aligned}
$$


A matriz [A] é uma matriz quadrada de ordem $2 n$ e a matriz [B] possui a dimensão $(2 n \times 1)$. A equação de estado mostrada em (18) serão resolvidas por meio do método de integração numérica de Heun que são implementadas com qualquer linguagem computacional. As oscilações espúrias são inerentes ao método numérico de integração utilizado para as equações de estado.

\section{RESULTADOS}

A Fig. 3 mostra uma linha de transmissão trifásica que será utilizada nas simulações deste trabalho. A linha será considerada transposta e com plano de simetria vertical, sendo as fases 1, 2 e 3 constituídas por 4 subcondutores do tipo Grosbeak, raio de 1,021 cm em cada fase, e os condutores $4 \mathrm{e}$ 5 são cabos para-raios do tipo $E H S W-3 / 8$ ".

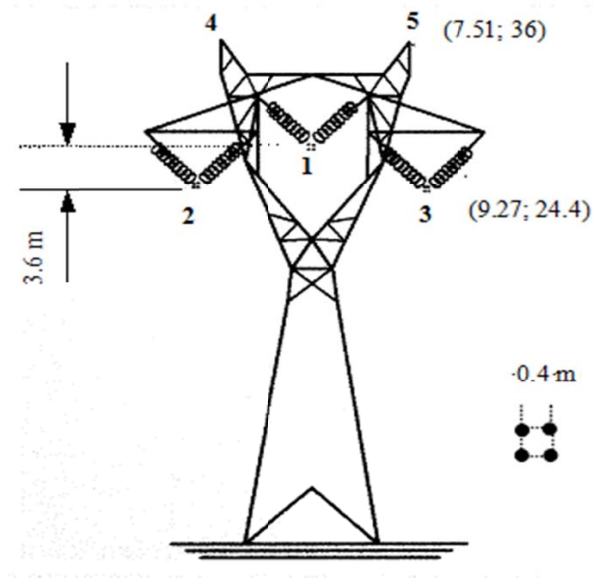

Figura 3. Linha trifásica utilizada nas simulações.

Os parâmetros longitudinais da linha foram calculados considerando os efeitos solo e pelicular. Considerando a linha mostrada na Fig. 5 são conhecidas para a frequência de $60 \mathrm{~Hz}$ as matrizes $\left[\mathrm{L}_{\text {linha }}\right](\mathrm{mH} / \mathrm{km})$ e $\left[\mathrm{C}_{\text {linha }}\right](\mathrm{nF} / \mathrm{km})$ são, respectivamente:

$$
\begin{aligned}
& {\left[L_{\text {linha }}\right]=\left[\begin{array}{lll}
0,8551 & 0,1217 & 0,1217 \\
0,1217 & 0,6340 & 0,0693 \\
0,1217 & 0,0693 & 0,6340
\end{array}\right]} \\
& {\left[C_{\text {linha }}\right]=\left[\begin{array}{lll}
14,6252 & -2,7246 & -2,7246 \\
-2,7246 & 19,8058 & -1,7830 \\
-2,7246 & -1,7830 & 19,8058
\end{array}\right]}
\end{aligned}
$$

Considerando o efeito skin e o efeito solo, as matrizes $\left[\mathrm{Z}_{\text {linha }}\right](\Omega / \mathrm{km})$ e $\left[\mathrm{Y}_{\text {linha }}\right](\mu \mathrm{S} / \mathrm{km})$, para a frequência de $60 \mathrm{~Hz}$, são dadas por:

$$
\left[Z_{\text {linha }}\right]=\left[\begin{array}{ccc}
0,6738+j 1,2263 & 0,0580+j 0,3430 & 0,0580+j 0,3430 \\
0,0580+j 0,3430 & 0,6740+j 1,1531 & 0,0581+j 0,3245 \\
0,0580+j 0,3430 & 0,0581+j 0,3245 & 0,6740+j 1,1531
\end{array}\right]
$$

$$
\left[Y_{\text {linha }}\right]=\left[\begin{array}{ccc}
j 0,5514 & -j 0,1027 i & -j 0,1027 \\
-j 0,1027 & j 0,7467 & -j 0,0672 \\
-j 0,1027 & -j 0,0672 & j 0,7467
\end{array}\right]
$$

Serão calculadas as tensões no terminal receptor em aberto para uma linha energizada por uma fonte de tensão senoidal trifásica e equilibrada. Utilizando a $\left[\mathrm{T}_{\text {clarke }}\right]$ a linha trifásica será decomposta em três linhas monofásicas independentes. Cada linha monofásica será representada pelos dois modelos descritos anteriormente. Verificam-se nas respostas do modelo a parâmetros discretos oscilações espúrias nas simulações. Serão mostradas as oscilações espúrias e feitas análises do seu conteúdo espectral considerando a linha representada por diferentes quantidades de circuitos $\pi$.

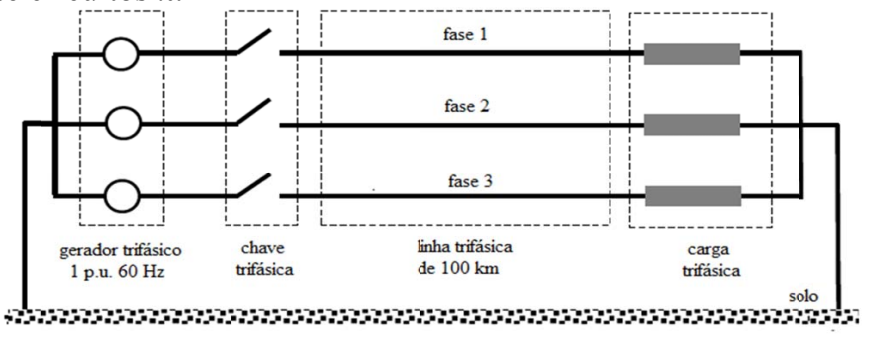

Figura 4. Linha trifásica com terminal receptor em aberto usado nas simulações.

A Fig. 5 mostra um modo de propagação genérico $m$ representado pelo modelo a parâmetros discretos.

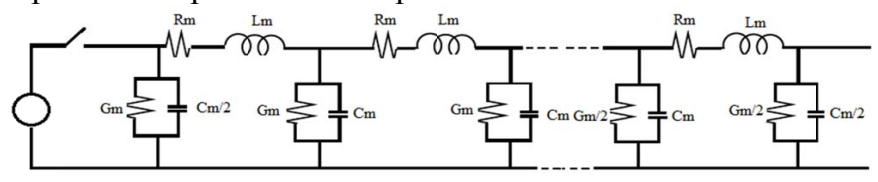

Figura 5. Linha genérica para o modo $\mathrm{m}$.

A Fig. 6 mostra o diagrama dos procedimentos para o desacoplamento da linha trifásica e cálculo das tensões modais e trifásicas (no domínio das fases).

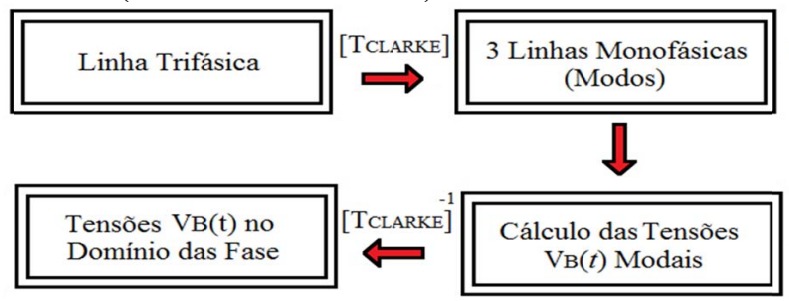

Figura 6. Procedimento para o calculo das tensões trifásicas modais.

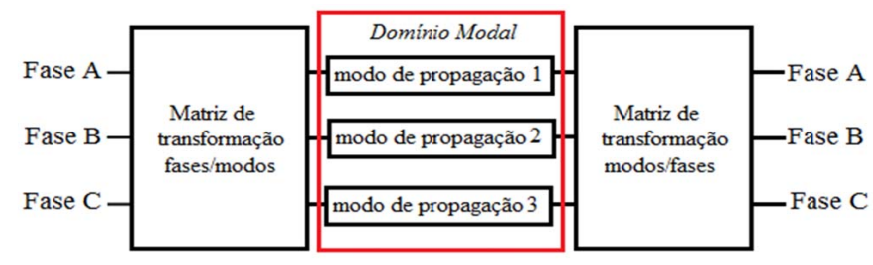

A tabela I mostra os parâmetros da linha de transmissão para cada modo de propagação $\mathrm{m}$ : 
Tabela I. PARÂMETROS ELÉTRICOS PARA CADA MODO M.

\begin{tabular}{lccc}
\hline \hline & & MODO & \\
PARÂMETROS & ALFA & BETA & ZERO \\
$\mathrm{R} m(\Omega / \mathrm{km})$ & 0,6159 & 0,6159 & 0,79 \\
$\mathrm{~L} m(\mathrm{mH} / \mathrm{km})$ & 2,3 & 2,2 & 4,9 \\
$\mathrm{C} m(\mathrm{nF} / \mathrm{km})$ & 0,1939 & 0,2159 & 0,13255 \\
$\mathrm{G} m(\mu \mathrm{S} / \mathrm{km})$ & 0 & 0 & 0
\end{tabular}

As tensões medidas no terminal receptor $\mathrm{B}$ em aberto, $\mathrm{V}_{\mathrm{B}}(\mathrm{t})$, considerando $\mathrm{o}$ modelo a parâmetros distribuídos podem ser observadas conforme a Fig. 7 .

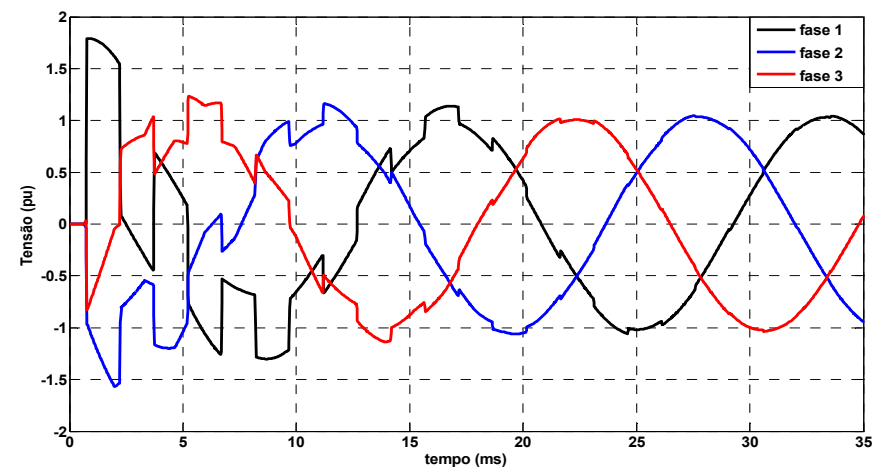

Figura 7. Tensões $V_{B}(t)$ considerando a linha representada pelo modelo a parâmetros distribuídos.

A linha trifásica foi representada pela quantidade de 5, 100 e 200 circuitos $\pi$ para verificar a influência da quantidade de circuitos $\pi$ no surgimento das oscilações espúrias nas simulações. As respostas serão comparadas com as simulações obtidas pelo ULM. A Fig. 8 mostra as tensões no terminal receptor B da linha de transmissão representada por 5 circuitos.

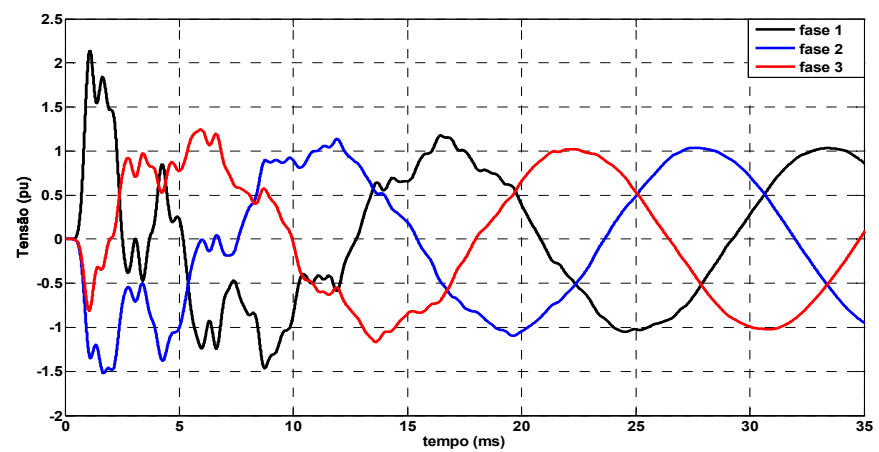

Figura 8. Tensões $\mathrm{V}_{\mathrm{B}}(\mathrm{t})$ considerando a linha representada por 5 circuitos $\pi$.

A Fig. 9 mostra as tensões no terminal receptor representada por 100 circuitos $\pi$.

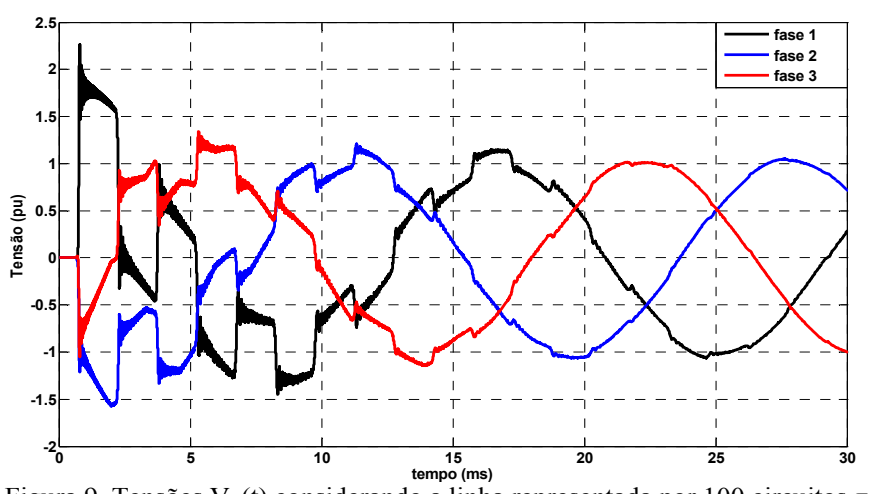

Figura 9. Tensões $V_{B}(t)$ considerando a linha representada por 100 circuitos $\pi$.

A Fig. 10 mostra as tensões no terminal receptor representada por 200 circuitos $\pi$.

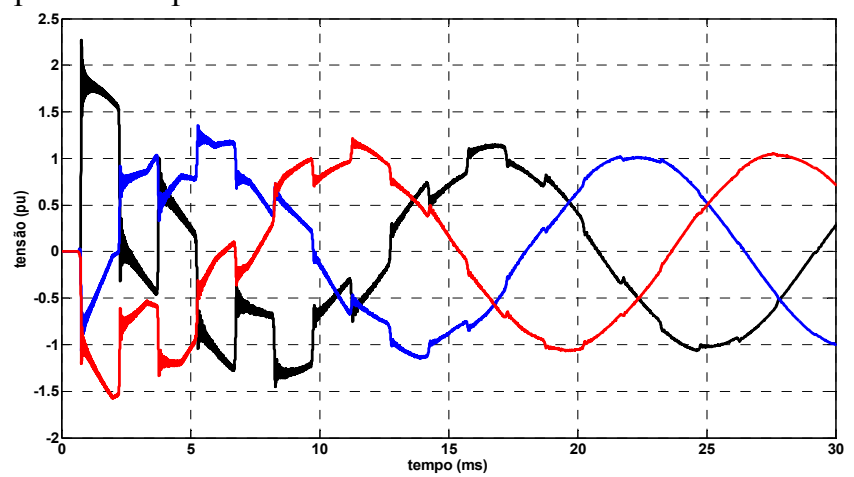

Figura 10. Tensões $\mathrm{V}_{\mathrm{B}}(\mathrm{t})$ para a linha representada por 200 circuitos $\pi$.

As Figs. 9 e 10 representam as tensões no terminal B adequadamente. Verifica-se que as respostas são semelhantes em todas as simulações, porém as Figs. 8 a 10 contêm oscilações espúrias. As oscilações ocorrerem devido à representação de um pequeno segmento de linha, cujos parâmetros são distribuídos, por cascata de circuitos $\pi$ que utiliza elementos discretos de circuitos e independem do método numérico utilizado para resolução das equações de estado [9]. Assim pode-se utilizar o modelo a parâmetros discretos para estudar transitórios eletromagnéticos em linhas de transmissão. As oscilações não representam o valor real da tensão transitória. Nas Figs. 9 e 10, considerando a primeira oscilação, essa está 30\% acima do valor esperado). Esse pico, caso considerado, pode resultar na atuação indevida de sistemas de proteção ou sobredimensionamento de isoladores durante seu projeto, tornando-o mais custoso. Diversos programas como $\mathrm{ATP}^{\circledR}, \mathrm{EMTP}^{\circledR}$, PSCAD $^{\circledR}$ usam o modelo a parâmetros discretos na representação de linhas e distintos métodos de integração trapezoidal e as oscilações podem ser observadas nos transitórios eletromagnéticos. Para estudar o conteúdo espectral das oscilações espúrias será aplicada a Transformada de Fourier nas simulações obtidas. Na Fig. 11 são mostradas as tensões $\mathrm{V}_{\mathrm{B}}(\mathrm{t})$ na fase 1 durante a primeira reflexão de onda para a linha de transmissão representada por $n$ circuitos $\pi$ em cascata, considerando um comprimento fixo de $100 \mathrm{~km}$. 


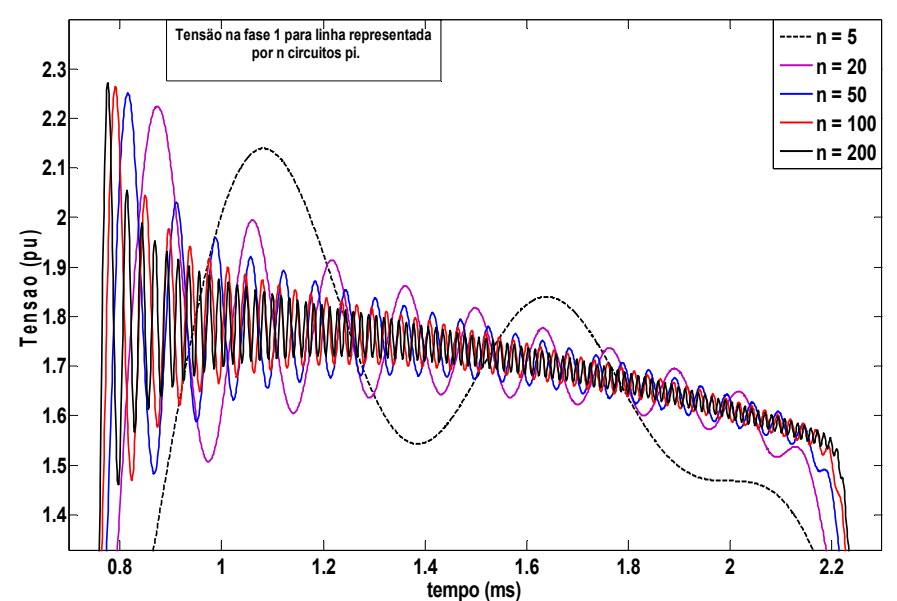

Figura 11. Tensões $V_{B}(t)$ considerando a linha representada $n$ circuitos $\pi$.

As oscilações espúrias dependem da quantidade de circuitos $\pi$ utilizada na representação da linha. Para as quantidades acima de $0,5 \pi / \mathrm{km}$, o transitório é mais bem representado. Os resultados também mostram que para 100 e 200 circuitos $\pi$ o transitório não apresenta uma diferença significativa. Isto sugere que ocorre uma saturação na resposta, para dado comprimento fixo da linha.

Mamis [13] calcula o erro obtido no cálculo da tensão $\mathrm{V}_{\mathrm{B}}(\mathrm{t})$ devido à representação da linha de transmissão monofásica por $\mathrm{n}$ circuitos $\pi$ com a resposta obtida para o modelo a parâmetros distribuídos. Nesse estudo a variação do erro percentual é inversamente proporcional com o número de circuitos $\pi$ usado. Mamis também propõe que para um dado erro tolerável, pode-se encontrar o número exato de circuitos $\pi$ para representar a linha, evitando o número excessivo de circuitos $\pi$ e os esforços computacionais e erros acumulativos são eliminados.

As Figs. 12 a 15 mostram os espectros de frequência das tensões no terminal em aberto com a linha representada por diversas quantidades de circuitos e em seguida será feita uma análise sobre os resultados obtidos. A Fig. 11 mostra o espectro de frequência das tensões obtido pelo modelo a parâmetros distribuídos.

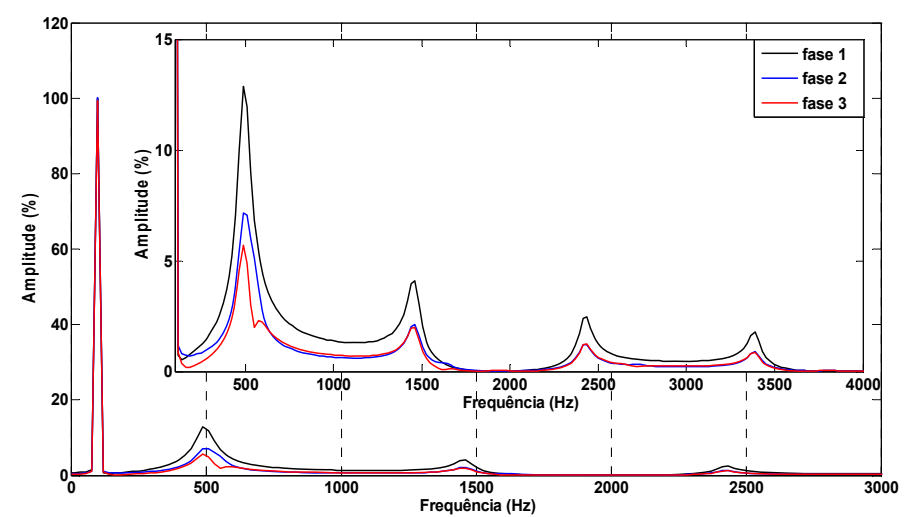

Figura 12. Espectro de $\mathrm{V}_{\mathrm{B}}(\mathrm{t})$ para o modelo a parâmetros distribuídos.
As Figs. 13-15 mostram o espectro das tensões $V_{B}(t)$ no terminal receptor em aberto obtidas com o modelo a parâmetros discretos com 5, 100 e 200 circuitos $\pi$.

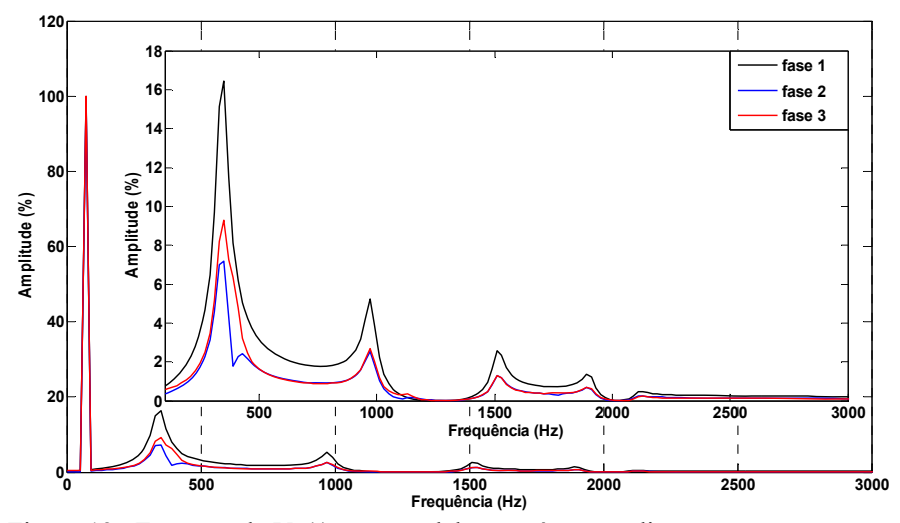

Figura 13. Espectro de $\mathrm{V}_{\mathrm{B}}(\mathrm{t})$ para modelo a parâmetros discretos com 5 circuitos $\pi$.

Na Fig. 12 mostra o espectro das tensões no terminal receptor da linha considerando o modelo a parâmetros distribuídos. As amplitudes são apresentadas em valores porcentuais (valor de base é a amplitude da componente fundamental das tensões). Assim por exemplo, a amplitude da componente do espectro de frequência para $500 \mathrm{~Hz}$ é equivalente a $13 \%$ da componente fundamental.

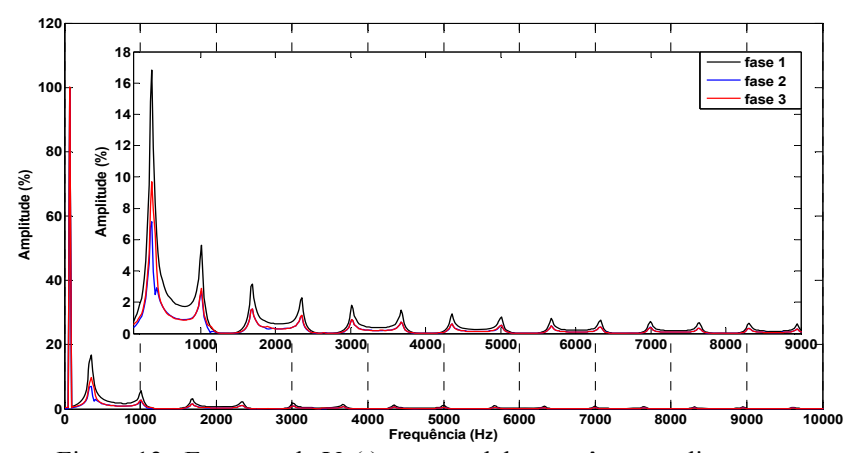

Figura 13. Espectro de $\mathrm{V}_{\mathrm{B}}(\mathrm{t})$ para modelo a parâmetros discretos com 100 circuitos $\pi$.

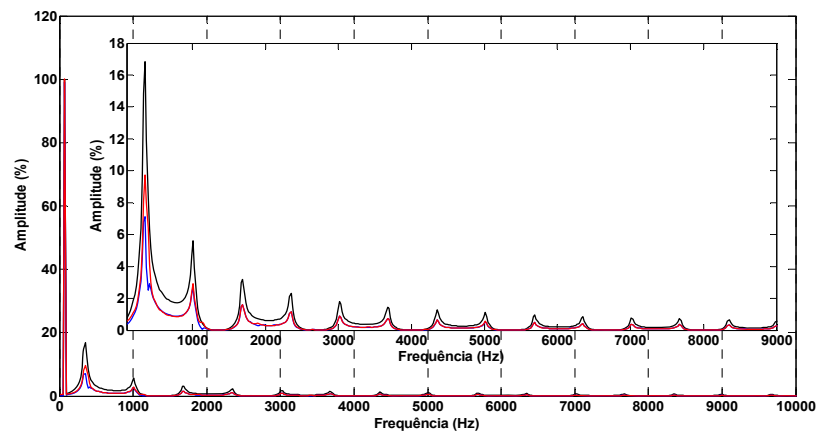

Figura 15. Espectro de $\mathrm{V}_{\mathrm{B}}(\mathrm{t})$ para modelo a parâmetros discretos com 200 circuitos $\pi$.

A quantidade de circuitos $\pi$ influencia no espectro de frequência. Para uma quantidade de 5 circuitos $\pi$, a tensão apresenta poucas componentes de elevadas frequências quando comparada com uma linha representada por 100 ou 
200 circuitos $\pi$. Para a linha representada com 100 circuitos $\pi$, a resposta contém maior conteúdo espectral, porém as amplitudes são inferiores quando comparadas com a Fig.10. Para o espectro da tensão com a linha representada por 200 circuitos $\pi$ verifica-se que não há diferenças significativas, sugerindo uma saturação na quantidade de circuitos $\pi$ a ser utilizada na representação de linhas de transmissão. Assim a quantidade de circuitos $\pi$ insere frequências na resposta e essas estão diretamente ligadas às oscilações espúrias observadas nas respostas no domínio do tempo.

\section{CONCLUSÕES}

Nesse trabalho a linha de transmissão trifásica foi representada por dois modelos de linha de transmissão para o cálculo das tensões no terminal receptor em aberto. A linha de transmissão trifásica foi representada por dois modelos. O primeiro modelo é o modelo a parâmetros discretos que considera a linha representada por uma cascata de circuitos $\pi \mathrm{e}$ desenvolvido diretamente no domínio do tempo. O outro modelo é denominado de o modelo a parâmetros distribuídos desenvolvido no domínio da frequência e em seguida aplica-se a Transformada Inversa de Laplace implementada numericamente, obtendo as resposta no domínio do tempo.

Observou-se a presença de oscilações espúrias em todas as simulações considerando o modelo a parâmetros discretos para a linha submetida a diversas condições de energização. Os resultados mostraram que o desempenho do modelo a parâmetros discretos dependem da quantidade de elementos discretos utilizados para representar a linha e que as oscilações espúrias são inerentes ao método de integração numérica usado para resolver as equações de estado.

Verifica-se no espectro de frequências das tensões trifásicas, considerando o comprimento de linha fixo, que a medida que se aumenta a quantidade de circuitos $\pi$, as amplitudes das componentes espectrais das oscilações espúrias são reduzidas e são inseridas mais componentes no seu espectro.

Os resultados também mostraram que ocorre saturação das oscilações presentes nas respostas com o aumento da quantidade de circuitos $\pi$ usados na representação de linhas de transmissão. Assim a quantidade de circuitos $\pi$ influência diretamente na resposta no domínio do tempo e na sua composição espectral, e devem sem levadas em consideração para o estudo de transitórios eletromagnéticos em linha de transmissão.

\section{AgRADECIMENTOS}

Os autotres agradecem pelo apoio da Fundação de Amparo à Pesquisa do Estado de São Paulo - FAPESP (2012/13857-4) e do Conselho Nacional de Desenvolvimento Científico e Tecnológico - CNPq.

\section{REFERÊNCIAS}

[1] S. Kurokawa, F. N. R. Yamanaka, A. J. Prado, J. Pissolato,"Representação de Linhas de Transmissão por meio de Variáveis de Estado Levando em Consideração o Efeito da Frequência sobre os Parâmetros Longitudinais", Revista Controle \& Automação, vol. 18, no 3, pp. 337-346, july, august , setember 2007
[2] Tavares, C., Pissolato J., Portela C.M., "Mode Domain Multiphase Transmission Line Model-Use in Transient Studies", IEEE Transactions on Power Delivery, vol. 14, no 4, pp. 1533-1544, october 1999.

[3] Marti, J. R., "Accurate Modelling of Frequency-Dependent Transmission Lines in Electromagnetic Transient Simulations", IEEE Transactions on Power Apparatus and Systems.vol. PAS-101. issue 1, pp.147-157, january 1982

[4] S. Kurokawa, R.C. SILVA, "Alternative model of three-phase transmission line theory-based modal decomposition", Revista IEEE América Latina, vol. 10, pp. 2074-2079, september 2012.

[5] B. Gustavsen, "Validation of Frequency Dependent Transmission Line Models",IEEE Transactions on Power Delivery, vol. 20, No 2, pp. 925 933, april 2005

[6] P. Moreno, A. Ramirez, "Implementation of the Numerical Laplace Transform: A Review”,IEEE Transactions on Power Delivery, vol. 23, No 4, pp. 2599-2609, october 2008.

[7] M. S. Mamis, "Computation of Electromagnetic Transients on Transmission Lines with Nonlinear Components", IEE Proceedings Generarion, Transmission and Distribution, v. 150, No 2, pp. 200-203, march 2003.

[8] S. Ma, B. Xu, Z. Bo, Klimberk A., "The Reaserch on Lumped Parameter Equivalent Circuit of Transmission Line" Advances in Power System Control, Operation and Management (APSCOM 2009), 8th International Conference, pp 1-5, november. 2009.

[9] A R. J. Araújo, R. C Silva, S. Kurokawa, "Representation of Transmission Lines: Comparison of Models and Parameters Distributed Discrete Parameters"; Revista IEEE América Latina, vol. 14,série 4, pp.1047-1052, june 2013.

[10] Budner, A. (1970) "Introduction of frequency-dependent line parameters into an electromagnetic transients program". IEEE Trans. Power App. And Systems, New York, vol. PAS-89, n. 1, pp. 88-97

[11] Moreno, P., Naredo J. L, Guardado J. L (2008). "Frequency domain transient analysis of electrical networks including non-linear conditions". International Journal of Electrical Power \& Energy Systems, vol. 27, n. 2, pp. 139-146.

[12] Dommel, H. W. (2007) "Digital Computer of Electromagnetic Transients in Single and Mutiphase Networks", IEEE Transactions on Power Apparatus and Systems, vol.PAS-88, n. 4, pp. 388-399.

[13] Mamis, M.S. (2010) "Remark on the Lumped Parameter Modeling of Transmission Lines", Electric Machines \& Power Systems, vol. 28, n. 6, pp. $565-575$.

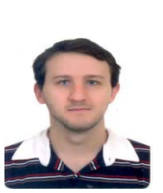

Anderson Ricardo Justo de Araújo Graduado (2012) e mestre (2014) em Engenharia Elétrica pela Faculdade de Engenharia Elétrica de Ilha Solteira, UNESP - Ilha Solteira e atualmente é estudante de doutorado em Engenharia Elétrica na Faculdade de Engenharia de Ilha Solteira, UNESP. Suas áreas de interesse são: transitórios eletromagnéticos em sistemas elétricos de potência utilizando métodos numéricos e modelagem de linhas de transmissão.

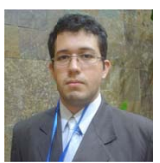

Rodrigo Cleber da Silva Graduado (2010) em mestre em engenharia elétrica pela Faculdade de Engenharia Ilha Solteira. Atualmente está no doutorado na mesma universidade. Professor no Instituto Federal de São Paulo (IFSP). Suas principais áreas de interesse são transitórios eletromagnéticos em sistemas de potência, modelos matemáticos para linhas de transmissão e métodos numéricos para análise transitória.

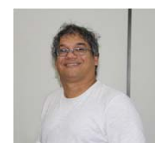

Sérgio Kurokawa (S'01-M'04) Graduado em Engenharia Elétrica (1990). Desde 1994 atua como Professor na Faculdade de Engenharia de Ilha Solteira da Universidade Estadual Paulista (UNESP). Obteve o título de Doutor em Engenharia Elétrica na Faculdade de Engenharia Elétrica e da Computação da Universidade Estadual Paulista (UNICAMP). Suas principais áreas de interesse são transitórios eletromagnéticos em sistemas elétricos de potência e modelos de linhas de transmissão para simulações de transitórios eletromagnéticos em sistemas de potência. 